\title{
Two-Way Denoise-And-Forward Relaying With Non-Coherent Differential Modulation
}

\author{
Wei Guan, K. J. Ray Liu \\ Department of Electrical and Computer Engineering \\ University of Maryland, College Park, MD 20742 \\ Email: \{wguan, kjrliu\}@umd.edu
}

\begin{abstract}
This work focuses on a two-way denoise-andforward relaying system using non-coherent Differential Binary Phase-Shift Keying (DBPSK) modulation. The relay denoising function and source decoders are designed using Maximum Likelihood (ML) principles. As the ML denoising function is hard to manipulate, it is approximated as a multi-user detector followed by a physical layer network coding encoder, based on which the closed-form relay decoding error is obtained. It is further shown that the ML source decoder is actually equivalent to the typical DBPSK decoder to the relay-source channel, and the exact end-to-end Bit Error Rate (BER) is derived then. A power allocation problem is also formulated to minimize the average BER at high Signal-to-Noise Ratio (SNR). It is shown that the optimal source power is inversely proportional to the square root of the channel gain of the source-relay channel, and the optimal relay power decreases with SNR.
\end{abstract}

\section{INTRODUCTION}

Cooperative communications, which can provide distributed spatial diversity and make a more efficient use of transmitted power [1], have gained a lot of research interests recently. The two widely discussed relaying protocols are Amplify-andForward (AF) and Decode-and-Forward (DF) [2], in which the relay node usually works in a half-duplex way. The spectral efficiency is thus low, as two time phases are required to deliver only one information unit, which introduces a pre-log factor $\frac{1}{2}$ on the spectral efficiency [3].

The Two-Way Relaying (TWR) system are thus proposed to fully recover the rate loss resulted from half-duplexing, as only 2 time phases, i.e., Broadcasting (BC) phase and Multiple Access (MA) phase, are required to assist the communication on both directions. Early work on AF-TWR and DF-TWR can be found in [3], which shows great improvements on sumrates. Later, [4] proposes a new Denoise-and-Forward (DNF) protocol, and a similar scheme called Physical-Layer Network Coding (PLNC) is proposed in [5], where the condition to guarantee one-to-one mapping is also given. Later work [6] shows that DNF-TWR has higher sum-rates than AF-TWR and DF-TWR, and [7] derives the closed-form BER of DNFTWR with coherent BPSK modulation.

While TWR opens a door to improve spectral efficiency, most of the existing work [4]-[8] assume that the terminals have full knowledge of Channel State Information (CSI), which is actually hard to acquire in a fast-fading environment [1]. In a limited number of literatures about TWR using noncoherent modulation, [9] designs the non-coherent decoder for minimum-shift keying signals and validates the throughput gain on a software radio testbed; [10] gives the symbol error rate for AF-TWR with relay selection; and [11] designs a set of non-coherent decoders for both AF-TWR and DNF-TWR using differential modulation.

As summarized above, DNF-TWR with non-coherent modulation can benefit from both the high spectral efficiency and reduced channel estimation overhead. However, to the best of our knowledge, the performance analysis and resource allocation scheme for such system is still an open problem, which motivates this work. Specifically, we focus on a DNFTWR system using non-coherent Differential BPSK (DBPSK) modulation. We first derive the relay denoising function and source decoder using Maximum Likelihood (ML) principles, and then proceed to analyze the corresponding decoding errors. As it is hard to manipulate the ML denoising function directly, we approximate it as a Multi-User Detector (MUD) followed by a PLNC encoder and obtain the closed-form relay decoding error. Next, we derive the exact end-to-end BER after showing the equivalence between the ML source decoder and the typical DBPSK decoder to the relay-source channel. Finally, we investigate the power allocation problem so as to minimize the average system BER using asymptotic analysis. Simulations justify our results.

Notations: Boldface lowercase letter a and boldface uppercase letter $\mathbf{A}$ represent vector in column form and matrix, respectively. $\|\mathbf{a}\|$ and $|\mathbf{A}|$ represent the Euclidean norm of a vector $\mathbf{a}$ and the determinant of a square matrix $\mathbf{A}$, respectively. $(\cdot)^{*},(\cdot)^{T}$ and $(\cdot)^{H}$ stand for conjugate, transpose and conjugate transpose, respectively. We shall use abbreviation i.i.d. for independent and identically distributed, and denote $Z \sim \mathcal{C N}\left(\mu, \sigma^{2}\right)$ as a circularly symmetric complex Gaussian random variable $Z$ with i.i.d. real part and imaginary part $\sim \mathcal{N}\left(\mu, \frac{\sigma^{2}}{2}\right)$. We define $\operatorname{sign}(x)=1$ if $x>0$ and 0 otherwise. Finally, the probability of an event $\mathcal{A}$ and the Probability Density Function (PDF) of a random variable $Z$ are denoted by $P(\mathcal{A})$ and $f(Z)$, respectively.

\section{SYSTEM MODEL}

Consider a narrow-band DNF-TWR system, where the two sources $S_{1}$ and $S_{2}$ want to exchange information through a single relay node. At the beginning of the MA phase, $S_{i}$ for $i=1,2$ first generates a sequence of i.i.d uncoded BPSK symbols $b_{i}(n) \in\{-1,1\}$ of length $L$, where $n=1,2, \ldots, L$ is 


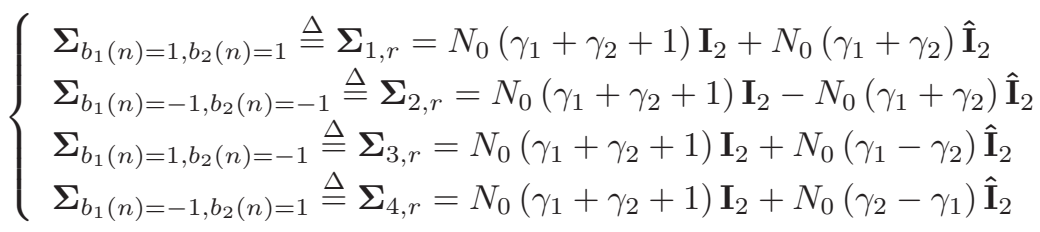

the symbol index. These raw symbols are then re-encoded through differential modulation, i.e., $x_{i}(n)=x_{i}(n-1) \times b_{i}(n)$ for $n=1,2, \ldots, L$ with $x_{i}(0)=1$ being the reference symbol. The two sources then send the whole block of differentially encoded symbols simultaneously to the relay during MA phase. To facilitate demonstrations, we define a sequence of auxiliary symbols $b(n)=b_{1}(n) \times b_{2}(n) \in\{-1,1\}$ for $n=1,2, \ldots, L$ to indicate whether the two raw BPSK symbols have the same signs or not. Note that because each source knows its own symbol, this common information $b(n)$ is sufficient for both sources to decode the symbol from the other end.

At the end of MA phase, the $n$th symbol received at the relay node is then

$$
y(n)=\sqrt{P_{s_{1}}} h_{1}^{M A} x_{1}(n)+\sqrt{P_{s_{2}}} h_{2}^{M A} x_{2}(n)+w^{M A}(n),
$$

where $P_{s_{i}}=\alpha_{i} P$ is the transmitted power of $S_{i}, P$ is the total power and $\alpha_{i} \in[0,1]$ stands for the corresponding power ratio. $h_{i}^{M A} \sim \mathcal{C N}\left(0, \sigma_{i}^{2}\right)$ is the channel coefficient from the $i$ th source to the relay during MA phase, where $\sigma_{i}^{2}$ is the channel gain. Here we assume that the channels remain unchanged within one block of length $(L+1)$; however, no terminals know such CSI so as to eliminate the channel estimation overhead. Finally, $w^{M A}(n) \sim \mathcal{C N}\left(0, N_{0}\right)$ is the Additive White Gaussian Noise (AWGN).

With DNF protocol[4][5], the relay just maps the $n$th receive symbol to another BPSK symbol $\hat{b}_{r}(n) \in\{-1,1\}$ that can be used by each source to uniquely decode the symbol transmitted from the other end. Here $\hat{b}_{r}(n) \in\{-1,1\}$ can be regarded as an estimate of the auxiliary symbol $b(n)$, so the relay denoising function is actually equivalent to the decoder for $b(n)$. As no CSI is available, we use the single-symbol ML decoder similar to that proposed in [11] throughout this work, i.e.,

$$
\hat{b}_{r}(n)=\arg \max _{b(n) \in\{-1,1\}} f(\mathbf{y}(n) \mid b(n)),
$$

where $\mathbf{y}(n)=(y(n), y(n-1))^{T}$ is the vector of two consecutive received symbols. It is easy to show that given $b_{1}(n)$ and $b_{2}(n),\left.\mathbf{y}(n)\right|_{b_{1}(n), b_{2}(n)} \sim \mathcal{C N}\left(\mathbf{0}, \boldsymbol{\Sigma}_{b_{1}(n), b_{2}(n)}\right)$, where the conditional covariance matrices are given in (3) on the top of this page. Here $\gamma_{i}=\frac{P_{s_{i}} \sigma_{i}^{2}}{N_{0}}=\alpha_{i} \sigma_{i}^{2} \gamma$ is the channel SNR from the $i$ th source to the relay, $\gamma=\frac{P}{N_{0}}$ is defined as the system SNR, and $\mathbf{I}_{2}=[1,0 ; 0,1]$ and $\hat{\mathbf{I}}_{2}=[0,1 ; 1,0]$ are two constant matrices. Based on the law of total probability, the conditional PDF of $\mathbf{y}(n)$ can be expressed as

$$
f(\mathbf{y}(n) \mid b(n))=\frac{1}{2} \sum_{b_{1}(n) \times b_{2}(n)=b(n)} f\left(\mathbf{y}(n) \mid b_{1}(n), b_{2}(n)\right) .
$$

After some manipulations, we have

$$
\hat{b}_{r}(n)=\operatorname{sign}(\ln (\operatorname{lrf}(\mathbf{y}(n) \mid b(n)))),
$$

where

$$
\operatorname{lr} f(\mathbf{y}(n) \mid b(n))=\frac{g\left(\mathbf{y}(n), \boldsymbol{\Sigma}_{1, r}\right)+g\left(\mathbf{y}(n), \boldsymbol{\Sigma}_{2, r}\right)}{g\left(\mathbf{y}(n), \boldsymbol{\Sigma}_{3, r}\right)+g\left(\mathbf{y}(n), \boldsymbol{\Sigma}_{4, r}\right)}
$$

is the Likelihood Ratio Function (LRF) of $\mathbf{y}(n)$, and

$$
g(\mathbf{y}, \boldsymbol{\Sigma})=\frac{1}{\pi^{2}|\boldsymbol{\Sigma}|} \exp \left(-\mathbf{y}^{H} \boldsymbol{\Sigma}^{-1} \mathbf{y}\right)
$$

is the PDF of $\mathbf{y} \sim \mathcal{C N}(\mathbf{0}, \boldsymbol{\Sigma})$. After decoding, the relay re-encodes $\left\{\hat{b}_{r}(n)\right\}_{n=1}^{L}$ into $t(n)=t(n-1) \times \hat{b}_{r}(n)$ for $n=1,2, \ldots, L$ through differential modulation with $t(0)=0$ being the reference symbol.

During BC phase, the relay broadcasts the symbols $t(n)$ to the source nodes. The received signal at $S_{i}$ is

$$
r_{i}(n)=\sqrt{P_{r}} h_{i}^{B C} t_{k}(n)+w_{i}^{B C}(n), n=0,1, \ldots, L,
$$

where $P_{r}=\beta P$ is the relay power and $\beta \in[0,1]$ is the corresponding power ratio. $h_{i}^{B C} \sim \mathcal{C N}\left(0, \sigma_{i}^{2}\right)$ is the channel coefficient from the relay to $S_{i}$ during $\mathrm{BC}$ phase, and we assume $h_{i}^{B C}$ and $h_{i}^{M A}$ are independent but have the same channel gain, which is determined by the distance between two terminals. $w_{i}^{B C}(n) \sim \mathcal{C N}\left(0, N_{0}\right)$ is the corresponding AWGN.

Each source only needs to detect $b(n)$ to decode the symbol from the other end. For example, if the decoded symbol for $b(n)$ at $S_{1}$ is $\hat{b}_{s_{1}}(n)=1$, then $b_{2}(n)$ can be decoded as $\hat{b}_{2, s_{1}}(n)=b_{1}(n)$, otherwise $\hat{b}_{2, s_{1}}(n)=-b_{1}(n)$ if $\hat{b}_{s_{1}}(n)=-1$. Again we assume $S_{i}$ uses the single-symbol ML decoder, i.e.,

$$
\hat{b}_{s_{i}}(n)=\arg \max _{b(n) \in\{-1,1\}} f\left(\mathbf{r}_{i}(n) \mid b(n)\right),
$$

where $\mathbf{r}_{i}(n)=\left(r_{i}(n), r_{i}(n-1)\right)^{T}$ comprises two consecutive received symbols, and $\left.\mathbf{r}_{i}(n)\right|_{\hat{b}_{r}(n)} \sim \mathcal{C N}\left(\mathbf{0}, \boldsymbol{\Sigma}_{\hat{b}_{r}(n), s_{i}}\right)$ with

$$
\left\{\begin{array}{l}
\boldsymbol{\Sigma}_{\hat{b}_{r}(n)=1, s_{i}} \triangleq \boldsymbol{\Sigma}_{1, s_{i}}=N_{0}\left(\bar{\gamma}_{i}+1\right) \mathbf{I}_{2}+N_{0} \bar{\gamma}_{i} \hat{\mathbf{I}}_{2} \\
\boldsymbol{\Sigma}_{\hat{b}_{r}(n)=-1, s_{i}} \triangleq \boldsymbol{\Sigma}_{2, s_{i}}=N_{0}\left(\bar{\gamma}_{i}+1\right) \mathbf{I}_{2}-N_{0} \bar{\gamma}_{i} \hat{\mathbf{I}}_{2}
\end{array},\right.
$$

where $\bar{\gamma}_{i}=\frac{P_{r} \sigma_{i}^{2}}{N_{0}}=\beta \sigma_{i}^{2} \gamma$ is the channel SNR from the relay to $S_{i}$. Now we can rewrite the joint PDF in (9) as

$$
f\left(\mathbf{r}_{i}(n) \mid b(n)\right)=\sum_{\hat{b}_{r}(n) \in\{-1,1\}} f\left(\mathbf{r}_{i}(n) \mid \hat{b}_{r}(n)\right) P\left(\hat{b}_{r}(n) \mid b(n)\right),
$$

where we use the law of total probability and the fact $\mathbf{r}_{i}(n)$ is independent with $b(n)$ conditioned on $\hat{b}_{r}(n)$. Based on (11), the ML source decoder (9) can be simplified to

$$
\hat{b}_{s_{i}}(n)=\operatorname{sign}\left(\ln \left(\operatorname{lrf}\left(\mathbf{r}_{i}(n) \mid b(n)\right)\right)\right),
$$




$$
\operatorname{lrf}\left(\mathbf{r}_{i}(n) \mid b(n)\right)=\frac{g\left(\mathbf{r}_{i}(n), \boldsymbol{\Sigma}_{1, s_{i}}\right)\left(1-P_{M, r}\right)+g\left(\mathbf{r}_{i}(n), \boldsymbol{\Sigma}_{2, s_{i}}\right) P_{M, r}}{g\left(\mathbf{r}_{i}(n), \boldsymbol{\Sigma}_{1, s_{i}}\right) P_{F, r}+g\left(\mathbf{r}_{i}(n), \boldsymbol{\Sigma}_{2, s_{i}}\right)\left(1-P_{F, r}\right)}
$$

$$
\operatorname{lr} f(\mathbf{y}(n) \mid b(n))=\frac{\left|\boldsymbol{\Sigma}_{3, r}\right|}{\left|\boldsymbol{\Sigma}_{1, r}\right|} \frac{\cosh \left(\frac{N_{0}\left(\gamma_{1}+\gamma_{2}\right)}{\left|\boldsymbol{\Sigma}_{1, r}\right|} \mathbf{y}^{H}(n) \hat{\mathbf{I}}_{2} \mathbf{y}(n)\right)}{\cosh \left(\frac{N_{0}\left(\gamma_{1}-\gamma_{2}\right)}{\left|\boldsymbol{\Sigma}_{3, r}\right|} \mathbf{y}^{H}(n) \hat{\mathbf{I}}_{2} \mathbf{y}(n)\right)} \times \exp \left(\frac{\left|\boldsymbol{\Sigma}_{1, r}\right|-\left|\boldsymbol{\Sigma}_{3, r}\right|}{\left|\boldsymbol{\Sigma}_{1, r}\right|\left|\boldsymbol{\Sigma}_{3, r}\right|} N_{0}\left(\gamma_{1}+\gamma_{2}+1\right)\|\mathbf{y}(n)\|^{2}\right)
$$

where $\operatorname{lr} f\left(\mathbf{r}_{i}(n) \mid b(n)\right)$ given in (13) on the top of this page is the LRF of $\mathbf{r}_{i}(n)$ conditioned on $b(n)$, and

$$
\begin{aligned}
P_{M, r} & =P\left(\hat{b}_{r}(n)=-1 \mid b(n)=1\right) \\
& =P(\operatorname{lr} f(\mathbf{y}(n) \mid b(n)) \leq 1 \mid b(n)=1) \\
P_{F, r} & =P\left(\hat{b}_{r}(n)=1 \mid b(n)=-1\right) \\
& =P(\operatorname{lr} f(\mathbf{y}(n) \mid b(n))>1 \mid b(n)=-1)
\end{aligned}
$$

are two kinds of conditional decoding error at the relay. The calculation of these two terms is postponed to the next section. Note that as both the relay decoder (2) and source decoder (9) depend only on the second-order statistics of all channels, which remain unchanged over time, the whole system can benefit from a great reduction on channel estimation overheads.

\section{PERFORMANCE ANALYSIS}

\section{A. Relay Decoding Error}

By use of the law of total probability, we can write the relay decoding error as

$$
P\left(\hat{b}_{r}(n) \neq b(n)\right) \triangleq P_{e, r}=\frac{P_{M, r}+P_{F, r}}{2},
$$

where $P_{M, r}$ and $P_{F, r}$ are two kinds of conditional decoding error defined in (14) and (15), and both of them are related with $\operatorname{lr} f(\mathbf{y}(n) \mid b(n))$. After substituting (7) into (6) and doing some manipulations, we have (17) on the top of this page, where $\cosh (x)=\frac{e^{x}+e^{-x}}{2}$ is the hyperbolic cosine function. As it is really hard to analyze the error probability based on the above LRF, we use the following approximation

$$
\cosh (x) \approx \frac{\max \left(e^{x}, e^{-x}\right)}{2}=\frac{e^{|x|}}{2},
$$

which is quite tight when $|x|$ is not too small. After such approximation, only exponential terms are left with the exponent being a quadratic form of $\mathbf{y}(n)$, which is analytically tractable.

After substituting (18) back into (17), we will arrive at

$$
\operatorname{lrf}(\mathbf{y}(n) \mid b(n)) \approx \frac{\max \left(g\left(\mathbf{y}(n), \boldsymbol{\Sigma}_{1, r}\right), g\left(\mathbf{y}(n), \boldsymbol{\Sigma}_{2, r}\right)\right)}{\max \left(g\left(\mathbf{y}(n), \boldsymbol{\Sigma}_{3, r}\right), g\left(\mathbf{y}(n), \boldsymbol{\Sigma}_{4, r}\right)\right)} .
$$

Now if we use (19) instead in (5), it is easy to see that this suboptimal decoder is actually a MUD

$$
\left(\hat{b}_{1, r}(n), \hat{b}_{2, r}(n)\right)=\arg \max _{b_{i}(n) \in\{-1,1\}} f\left(\mathbf{y}(n) \mid b_{1}(n), b_{2}(n)\right)
$$

followed by a PLNC encoder $\hat{b}_{r}(n)=\hat{b}_{1, r}(n) \times \hat{b}_{2, r}(n)$.That is, the relay first jointly decodes the BPSK symbols $b_{1}(n)$ and $b_{2}(n)$, and then maps the decoded symbols to a single BPSK symbol $\hat{b}_{r}(n)$ as an estimate of the indicator symbol $b(n)$. As we shall see in simulations, this suboptimal relay decoder works almost as well as the ML decoder (5) in all cases.

After some manipulations, we can show that

$$
\begin{aligned}
P_{M, r} & =h\left(u_{1}, u_{2}, a, b, \gamma_{t h}\right), \\
P_{F, r} & =1-h\left(u_{3}, u_{4}, a, b, \gamma_{t h}\right),
\end{aligned}
$$

where $u_{1}=\frac{1}{N_{0}\left(2 \gamma_{1}+2 \gamma_{2}+1\right)}, u_{2}=\frac{1}{N_{0}}, u_{3}=\frac{1}{N_{0}\left(2 \gamma_{1}+1\right)}$, $u_{4}=\frac{1}{N_{0}\left(2 \gamma_{2}+1\right)}$, and

$$
\begin{gathered}
a=-\frac{4 \gamma_{1} \gamma_{2}\left(\gamma_{1}+\gamma_{2}+1\right)}{N_{0}\left(2 \gamma_{1}+2 \gamma_{2}+1\right)\left(2 \gamma_{1}+1\right)\left(2 \gamma_{2}+1\right)} \\
b=\frac{4 \gamma_{1} \gamma_{2}\left(\gamma_{1}+\gamma_{2}\right)+2 \min \left(\gamma_{1}, \gamma_{2}\right)\left(2 \gamma_{1}+2 \gamma_{2}+1\right)}{N_{0}\left(2 \gamma_{1}+2 \gamma_{2}+1\right)\left(2 \gamma_{1}+1\right)\left(2 \gamma_{2}+1\right)} \\
\gamma_{t h}=\frac{\left(2 \gamma_{1}+2 \gamma_{2}+1\right)}{\left(2 \gamma_{1}+1\right)\left(2 \gamma_{2}, k+1\right)} \\
h\left(t_{1}, t_{2}, a, b, \gamma\right)=\frac{4 a b t_{1} t_{2} \exp \left(-\frac{t_{1}+t_{2}}{2 a} \ln \gamma\right)}{a^{2}\left(t_{1}-t_{2}\right)^{2}-b^{2}\left(t_{1}+t_{2}\right)^{2}} .
\end{gathered}
$$

Finally, plugging (21) and (22) back into (16) leads to the closed-form relay decoding error.

\section{B. Source Decoding Error}

After some manipulations, the source decoder (12) can be reduced to

$$
\begin{aligned}
\hat{b}_{s_{i}}(n) & =\operatorname{sign}\left(\ln \left(\operatorname{lrf}\left(\mathbf{r}_{i}(n) \mid b(n)\right)\right)\right) \\
& =\operatorname{sign}\left(\ln \left(\operatorname{lrf}\left(\mathbf{r}_{i}(n) \mid \hat{b}_{r}(n)\right)\right)\right) \triangleq \hat{b}_{r, s_{i}}(n),(27)
\end{aligned}
$$

where

$$
\operatorname{lrf}\left(\mathbf{r}_{i}(n) \mid \hat{b}_{r}(n)\right)=\frac{g\left(\mathbf{r}_{i}(n), \mathbf{\Sigma}_{1, s_{i}}\right)}{g\left(\mathbf{r}_{i}(n), \mathbf{\Sigma}_{2, s_{i}}\right)}
$$

is the LRF of $\mathbf{r}_{i}(n)$ conditioned on $\hat{b}_{r}(n)$. Note that the decoder on the second line of (27) is actually a typical noncoherent DBPSK decoder [12, Eqn.(14-4-23)] to the relaysource channel, whose output $\hat{b}_{r, s_{i}}(n)$ is an estimate of the decoded symbol $\hat{b}_{r}(n)$ at the relay. Using such equivalence, we can write the source decoding error as

$$
\begin{aligned}
P\left(\hat{b}_{s_{i}}(n) \neq b(n)\right) & =P\left(\hat{b}_{r, s_{i}}(n) \neq b(n)\right) \\
& \triangleq P_{e, s_{i}}=\frac{1}{2}\left(P_{M, s_{i}}+P_{F, s_{i}}\right),
\end{aligned}
$$


where

$$
\begin{aligned}
P_{M, s_{i}} & =P\left(\hat{b}_{r, s_{i}}(n)=-1 \mid b(n)=1\right), \\
P_{F, s_{i}} & =P\left(\hat{b}_{r, s_{i}}(n)=1 \mid b(n)=-1\right)
\end{aligned}
$$

are two kinds of conditional decoding error at $S_{i}$, and we use the relation $\hat{b}_{s_{i}}(n)=\hat{b}_{r, s_{i}}(n)$. After expanding (30) by use of the law of total probability, we have

$$
P_{M, s_{i}}=P_{D, s_{i}}\left(1-P_{M, r}\right)+\left(1-P_{D, s_{i}}\right) P_{M, r},
$$

where we use the fact that $\hat{b}_{r, s_{i}}(n)$ is independent of $b(n)$ conditioned on $\hat{b}_{r}(n)$, and that the two kinds of conditional decoding error of a typical non-coherent DBPSK decoder are equal and are given by [12, Eqn.(14-4-26)]

$$
\begin{aligned}
& P\left(\hat{b}_{r, s_{i}}(n)=1 \mid \hat{b}_{r}(n)=-1\right) \\
& \quad=P\left(\hat{b}_{r, s_{i}}(n)=-1 \mid \hat{b}_{r}(n)=1\right) \\
& \quad \triangleq P_{D, s_{i}}=\frac{1}{2\left(\bar{\gamma}_{i}+1\right)} .
\end{aligned}
$$

In a similar way, we can derive

$$
P_{F, s_{i}}=\left(1-P_{D, s_{i}}\right) P_{F, r}+P_{D, s_{i}}\left(1-P_{F, r}\right) .
$$

Plugging (32) and (34) back into (29) we have

$$
P_{e, s_{i}}=\left(1-P_{D, s_{i}}\right) P_{e, r}+P_{D, s_{i}}\left(1-P_{e, r}\right),
$$

which is the end-to-end BER at $S_{i}$.

\section{POWER ALLOCATION}

Now we are about to investigate the power allocation among the two sources and the single relay so as to minimize the average BER, which can be formulated as

$$
\begin{gathered}
\min P_{e}=\frac{1}{2}\left(P_{e, s_{1}}+P_{e, s_{2}}\right) \\
\text { s.t. } \alpha_{1}+\alpha_{2}+\beta=1, \\
0 \leq \alpha_{1}, \alpha_{2}, \beta \leq 1 .
\end{gathered}
$$

However, it is hard to manipulate the exact BER expression (35) directly, and the optimal solution can only be derived through exhaustive search. In order to obtain one simple closed-form solution, we examine the asymptotic BER at high SNRs (i.e., $\gamma \gg 1$ ) and have

$$
\left\{\begin{array}{l}
P_{M, r} \approx \frac{c_{M, r}}{\gamma}, c_{M, r}=\frac{1}{2 \min \left(\alpha_{1} \sigma_{1}^{2}, \alpha_{2} \sigma_{2}^{2}\right)} \\
P_{F, r} \approx \frac{d_{F, r}}{\gamma} \ln \frac{\gamma}{d_{F, r}}, d_{F, r}=\frac{\alpha_{1} \sigma_{1}^{2}+\alpha_{2} \sigma_{2}^{2}}{2 \alpha_{1} \alpha_{2} \sigma_{1}^{2} \sigma_{2}^{2}} \\
P_{D, s_{i}} \approx \frac{q_{D, s_{i}}}{\gamma}, q_{D, s_{i}}=\frac{1}{2 \beta \sigma_{i}^{2}}, i=1,2
\end{array} .\right.
$$

After plugging these approximations back into (35), we have

$$
P_{e} \approx \frac{1}{2 \gamma}\left(c_{M, r}+d_{F, r} \ln \frac{\gamma}{d_{F, r}}+q_{D, s_{1}}+q_{D, s_{2}}\right),
$$

where we neglect the higher-order terms. There are several observations here. Firstly, it is easy to see that the BER is dominated by $P_{F, r}$, which scales as $\gamma^{-1} \ln \gamma$ at high SNRs. Therefore, more power should be allocated to the sources in order to reduce the relay decoding error. Secondly, the
BER of the direct transmission with non-coherent DBPSK modulation scales as $\gamma^{-1}$ [12, Eqn.(14-4-28)], which decreases faster than the dominant error term $P_{F, r}$ at high SNRs. In other words, the DNF-TWR is comparatively not preferred than direct transmission when SNR is increasing. Finally, it can be observed that $P_{F, r}>P_{M, r}$ when source power is fixed and SNR is sufficiently high. This is because it is relatively easier to decode $b(n)$ when the two source symbols have the same signs, in which case the two consecutive observations $y(n)$ and $y(n-1)$ would have similar envelopes at high SNRs.

Note that the first two terms in (38) depend only on $\alpha_{1}$ and $\alpha_{2}$ while the last two terms depend only on $\beta$. So (36) can be resolved by two steps. In the first step, we fix $\beta$ and seek to find the optimal source power, i.e.,

$$
\begin{array}{ll}
\min & \frac{c_{M, r}+d_{F, r} \ln \frac{\gamma}{d_{F, r}}}{2 \gamma} \approx \frac{d_{F, r}}{2 \gamma} \ln \frac{\gamma}{d_{F, r}} \\
\text { s.t. } & \alpha_{1}+\alpha_{2}=1-\beta, \\
& 0 \leq \alpha_{1}, \alpha_{2} \leq 1-\beta .
\end{array}
$$

where we neglect the term $c_{M, r}$ because it is much smaller than $\ln \gamma$ at high SNRs. Note that the function $\phi(x)=x \ln x$ is increasing when $x<e^{-1}$, which is the case for sufficiently large $\gamma$. Therefore, it is equivalent to minimizing $d_{F, r}$ instead, whose optimizer is

$$
\left\{\begin{array}{l}
\alpha_{1}^{o p t}=(1-\beta) \frac{\sigma_{2}}{\sigma_{1}+\sigma_{2}} \\
\alpha_{2}^{o p t}=(1-\beta) \frac{\sigma_{1}}{\sigma_{1}+\sigma_{2}}
\end{array} .\right.
$$

Clearly, the optimal source power is inversely proportional to the square root of the channel gain of the corresponding source-relay channel. That is, more power should be allocated to the source that is far away from the relay, otherwise its signal would be shadowed by that from the other end during MA phase, which increases the relay decoding error. Therefore, the above source power allocation scheme actually provides an elegant way to resolve the near-far problem. Next, if we plug (40) into (38), it leads to an optimization problem that only involves the relay power coefficient $\beta$, i.e.,

$$
\min \frac{\eta_{1}}{1-\beta}+\frac{\eta_{2}}{\beta} \text {, s.t. } 0 \leq \beta \leq 1,
$$

where

$$
\begin{aligned}
\eta_{1}= & \frac{\sigma_{1}+\sigma_{2}}{4 \gamma \sigma_{1} \sigma_{2} \min \left(\sigma_{1}, \sigma_{2}\right)} \\
& \quad+\frac{\left(\sigma_{1}+\sigma_{2}\right)^{2}}{4 \gamma \sigma_{1}^{2} \sigma_{2}^{2}} \ln \frac{2 \gamma \sigma_{1}^{2} \sigma_{2}^{2}}{\left(\sigma_{1}+\sigma_{2}\right)^{2}}, \\
& \\
\eta_{2}= & \frac{\sigma_{1}^{2}+\sigma_{2}^{2}}{4 \gamma \sigma_{1}^{2} \sigma_{2}^{2}} .
\end{aligned}
$$

Note that we neglect the term $(1-\beta)$ within the log function in (42) when deriving the objective function in (41), as it is generally much smaller than $\gamma$ at high SNRs. The optimizer of (41) can be easily derived as

$$
\beta^{o p t}=\frac{\sqrt{\eta_{2}}}{\sqrt{\eta_{1}}+\sqrt{\eta_{2}}} .
$$




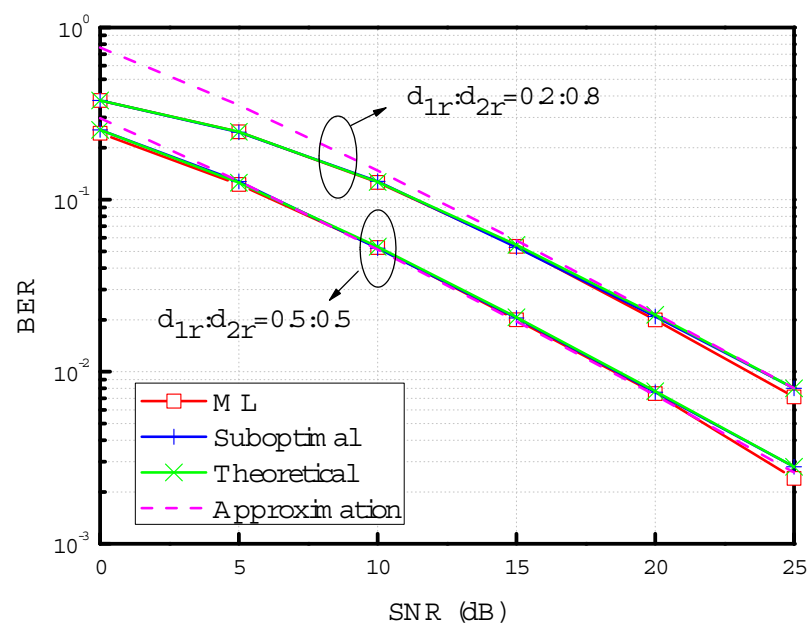

Fig. 1. BER performances versus SNR.

It can be shown that $\beta^{o p t}$ is a decreasing function with SNR, which coincides with our previous analysis that more power should be allocated to the source as SNR is increasing.

\section{NUMERICAL RESULTS}

In this section, we shall present some simulation results for the considered system. We use the path loss model $\sigma^{2}=d^{-4}$, where $\sigma^{2}$ is the channel gain and $d$ is the distance between two terminals. For simplicity, we normalize the distance between two sources to 1 , and we always place the relays on the line connecting two sources. In all cases, BER refers to the average decoding error at source 1 and source 2. Without special explanation, the transmitted power is always equally split among all terminals.

We first examine the BER performance in Fig. 1 , where $d_{1, r}$ and $d_{2, r}$ are the distances between the relay and two sources, respectively. We compare the simulated BER of different relay decoders with the theoretical results. The suboptimal relay decoder refers to the MUD followed by a PLNC encoder. It can be observed that there is almost no difference between the ML decoder and the suboptimal one, and both of them coincide with our theoretical results. Besides, the asymptotic BER is tight when SNR is sufficiently high, e.g., when $\gamma \geq 15 d B$ for $d_{1, r}: d_{2, r}=0.2: 0.8$ and when $\gamma \geq 5 d B$ for $d_{1, r}: d_{2, r}=0.5: 0.5$. The tightness for the latter case is due to the high channel gains of both of the two source-relay channels, which makes it easier to satisfy the high SNR assumption.

Then in Fig. 2, we proceed to study the performance gain of power allocation. The optimal scheme is found through exhaustive search, and the suboptimal one refers to that derived through asymptotic analysis. Compared with equal power allocation, about 2dB SNR gain can be observed in Fig. 2 when $d_{1, r}: d_{2, r}=0.1: 0.9$. Such performance gain is diminishing as the relay moves to the halfway between two sources, in which case the equal power allocation is near-optimal.

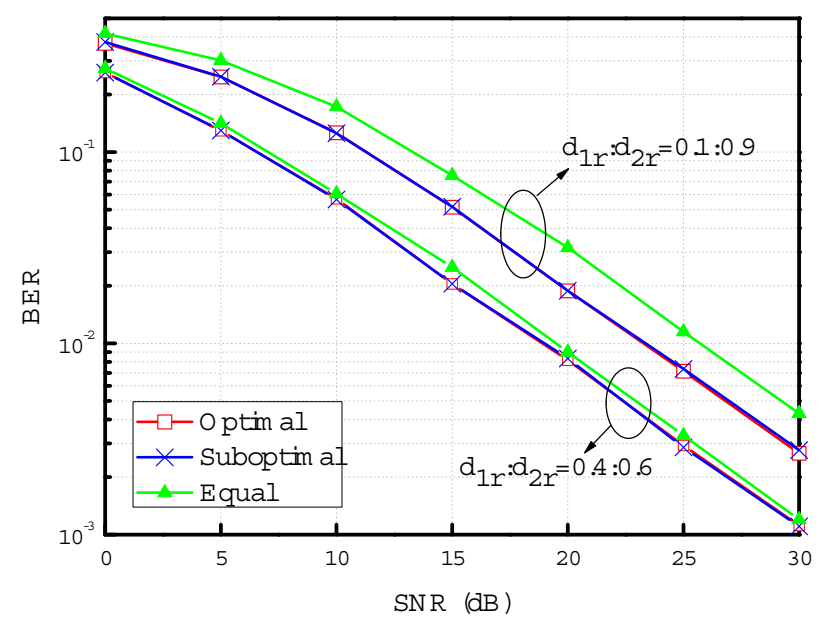

Fig. 2. BER performances with power allocation versus SNR.

\section{CONCLUSION AND FUTURE WORK}

In this work, we have analyzed the BER performances of the DNF-TWR system using non-coherent DBPSK modulation. A near-optimal power allocation scheme is also derived based on the asymptotic analysis at high SNRs. Future work may focus on the system with multiple relays. One may also investigate the denoising function design using higher-order non-coherent modulations.

\section{REFERENCES}

[1] K. J. R. Liu, A. K. Sadek, W. Su, and A. Kwasinski, Cooperative Communications and Networking, Cambridge Univ. Press, 2008.

[2] J. N. Laneman, D. N. C. Tse, and G. W. Wornell, "Cooperative Diversity in Wireless Networks: Efficient Protocols and Outage Behavior," IEEE Trans. Inf. Theory, vol. 50, no. 12, pp. 3062-3080, Dec. 2004.

[3] B. Rankov and A. Wittneben, "Spectral Efficient Protocols for HalfDuplex Fading Relay Channels," IEEE J. Sel. Areas Commun., vol. 25, no. 2, pp. 379-389, Feb. 2007.

[4] P. Popovski and H. Yomo,"The Anti-Packets Can Increase the Achievable Throughput of a Wireless Multi-Hop Network," in Proc. IEEE International Conference on Communication (ICC 2006), Istanbul, Turkey, pp. 3885-3890, Jun. 2006.

[5] S. Zhang, S. C. Liew, and P. P. Lam, "Physical-Layer Network Coding," in ACM MOBICOM 2006, Los Angeles, Sept. 2006.

[6] P. Popovski and H. Yomo, "Physical Network Coding in Two-Way Wireless Relay Channels", in Proc. IEEE International Conference on Communication (ICC 2007), Glasgow, Scotland, pp. 707-712, June, 2007.

[7] E. C. Y. Peh, Y.-C. Liang, and Y. L. Guan, "Power Control for Physical-Layer Network Coding in Fading Environments," in Proc. IEEE Personal, Indoor and Mobile Radio Commun. (PIMRC), Cannes, France, pp. 1-5, Sep.2008.

[8] P. Popovski and H. Yomo, "Wireless Network Coding by Amplify-andForward for Bi-directional Traffic Flows", IEEE Commun. Lett., Vol. 11, No. 1, pp. 16-19, Jan. 2007.

[9] S. Katti, S. Gollakota, and D. Katabi, "Embracing wireless interference: Analog network coding," in ACM SIGCOMM 2007, Aug. 2007.

[10] L. Y. Song, G. Hong, B. L. Jiao, and M. Debbah, "Joint Relay Selection and Analog Network Coding Using Differential Modulation in Two-Way Relay Channels," IEEE Trans. Veh. Technol., vol. 59, no. 6, pp. 29322939, July 2010.

[11] T. Cui, F. F. Gao, and C. Tellambura, "Differential Modulation for TwoWay Wireless Communications: A Perspective of Differential Network Coding at the Physical Layer," IEEE Trans. Commun., vol. 57, no. 10, pp. 2977-2987, Oct. 2009.

[12] J. Proakis, Digital Communications, 4th ed. New York: McGraw-Hill, 2001. 